\title{
EMU OIL, A NATURAL POLYVALENT THERAPY
}

\section{Dr. Maria Elena Nedea, Dr. Constantin Nedea,}

\author{
ChemPlan Consulting Inc., Montreal, 4428 Claude Henri Grignon, H4R3C3, Quebec, \\ Canada, chemplan@videotron.ca
}

\begin{abstract}
This paper is an endeavour to bring together some the research results and information available in the scientific literature, regarding the properties and the therapeutic uses of emu oil. The review was done with the purpose to inform readers about the beneficial effects of emu oil on one's health and to explain these effects based on its chemical composition. The Emu is a bird indigenous to Australia, belonging to the same family as the ostrich; from its fat, an oil is obtained through a process of melting and refining. The native people of Australia, the Aborigines, discovered thousands of years ago the benefits of emu oil and incorporated it into their culture as a valuable commodity. They used emu oil for a multitude of purposes: as a treatment for burns and arthritis, for improvement of muscular pain and as a remedy for skin ailments. Prior to 1990, very little research had been done on the emu and its oil in the US and Australia; however today, due to ongoing interest in the latter, there are a lot of research findings on the chemical composition, the properties and the therapeutic usage of emu oil. Frequently, numerous physicians recommending the treatment with emu oil to reduce the bad cholesterol level, or to ameliorate the symptoms of eczema, psoriasis, haemorrhoids, acne, gave evidence about its miraculous effects. Emu oil proved to be a nutraceutical and cosmeceutical product with beneficial effects on the health; it does not produce allergies or other adverse effects.
\end{abstract}

Keywords: Chemistry, Natural Products, Medicine

MOTTO

''Emu oil is an untapped resource and currently now is where aspirin was in the early 1900'.' Dr. Barry Sears, President of the Foundation for Research of Inflammation, USA

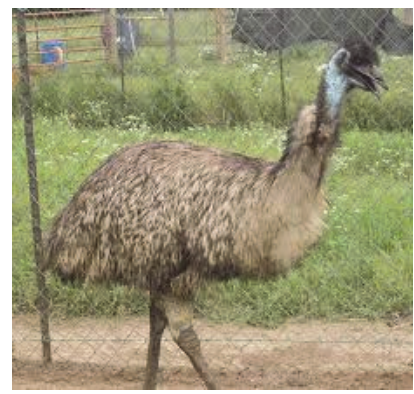

Figure 1. Emu bird

\section{The Emu and emu oil}

The emu bird (Dromaius novaehollandiae), shown in Figure 1 belongs to a group of birds known as "ratites", and is the second largest bird in the world behind its African ratite cousin, the ostrich. In $1960 \mathrm{emu}$ was declared the national bird of Australia, playing an important role in the life of Australian Aborigines.

The emu contributed to the survival of tribes of native Australian in the form of both food and medicine. Emu meat and eggs were a great source of lean protein and the oil rendered from its fat was used by Aborigines as a remedy for skin aliments, as an arthritis and burn treatment, as a skin protectant and sunscreen and as a skin moisturizer. The Aboriginal method for trauma treatment was to wrap the affected area with emu skin, fat side down, and put the patient in the sun, so the heat melts the fat and drive the oil into the skin.

Currently, emu oil is produced comercially by processing the roughly $10 \mathrm{~kg}$ of fat on a mature bird with heat and filtration, to give 5-6 litters of rendered oil. Therapeutic properties can vary depending on the diet and lifestyle of bird, with oil from feral birds presenting the best fatty acid profile, most efficacy in reduction of arthritic swelling, and an analgesic quality comparable to that of naproxen [1]. 


\section{The Properties of Emu Oil}

\subsection{Physical Properties}

The physical properties of emu oil are shown in Table 1.

Table 1. Physical properties of emu oil

$\begin{array}{ll}20^{\circ} \mathrm{C} & \text { Semisolid } \\ 60^{\circ} \mathrm{C} & \text { Liquid } \\ \text { Odor } & \text { Absent } \\ \text { Umidity } & 0,05 \% \\ \text { Refractive index } & 1,4654\left(30^{\circ} \mathrm{C}\right) \\ \text { Iodine value } & 75-80 \\ \text { Density } & 0,921 \mathrm{~g} / \mathrm{ml}\end{array}$

\subsection{Chemical Composition}

The therapeutic and cosmetic properties of emu oil are due to the presence in the composition of a high percentage $(70 \%)$ of the unsaturated fatty acids which have an important physiological effect. The major acid is the oleic acid (Omega 9), which is a known enhancer for transport of bioactive compounds into the skin, and thus the ability to be absorbed quickly and penetrate deeply when applied topically. Other two essential fatty acids which are highly important are the linoleic acid (Omega 6) and the linolenic acid (Omega 3) .

Table 2 indicates the chemical composition of emu oil [2].

Table 2. Chemical composition of emu oil

$\begin{array}{lc}\text { Acid gras } & \mathbf{\%} \\ \text { Palmitic (C16: 0) } & 22,0 \\ \text { Linoleic (C18: 2) } & 15,2 \\ \text { Stearic (C18: 0) } & 9,2 \\ \text { Oleic (C18: 1) } & 47,4 \\ \text { Palmitoleic (C16: 1) } & 3,1 \\ \text { Linolenic (C18: } 3) & 1,0\end{array}$

The overall composition of emu oil is very similar to our skin's own fatty acid composition, explaining the compatibility of the emu oil with the skin and its beneficial effect on the skin. Dr. Hopkins mentioned that one of the difficulties of the emu oil industry is to scientifically explain the ability of emu oil to relieve the symptoms of different diseases [3]. He specified that the proportion of fat in this oil or the ratio of saturated and unsaturated acids have a more important role in the therapeutic process than the individual presence of each acid (linoleic, oleic, palmitic, stearic, palmitoleic). These acids also exist in other oils, which do not possess the beneficial properties of the emu oil.

Dr. Sears, the President of the Foundation for Research of Inflammation, USA, has launched the hypothesis that emu oil contains a "factor $X^{\prime \prime}$ to which its "miraculous" properties are attributed. It modulates the eicosynoid hormones that control the immune system, central nervous system and other systems in the body [4]. Properly refined, emu oil is penetrating, anti-inflammatory, antibacterial, healing, moisturizing and noncomedogenic.

\section{Therapeutic Usage of Emu Oil}

\subsection{General Clinic Results}

Dr. Hobday, an Australian physician, was the pioneer of the clinical studies on emu oil. He published in 1994 a global report, containing the results of his clinical experience during 10 years, following the administration of emu oil on 500 patients [5].

The overall results obtained from the treatment were summarized by the researcher as follows:

\section{Skin}

- Eczema: Emu oil and creams containing emu oilreduce irritation and inflammation of the skin and significantly reduces tissue growth over a scar caused by a wound or a surgical incision.

- Burns: Faster healing, reducing pain and scarring.

- Skin transplant: reduces pain felt by removing the skin.

- Psoriasis: improvement in some patients.

\section{Joints}

-Joint pain: reduces pain, swelling and stiffness, especially when the joint is closer to the surface ofthe skin, such as the hand, foot, knee and shoulder.

- Rheumatoid arthritis: Dr. Hobday has not found any improvements. Considering that some of his colleagues have achieved positive results, he recommended continuing clinical trials. 
- Contusions and muscle pain: an important effect on recent contusions and muscle pain when the wound was relative to the surface of the skin. Emu oil massages are effective for relaxing strained muscles after a sporty exercise.

\section{Recent wounds}

- In skin-covered wounds, emu oil reduces tissue formation that results in scar enlargement and cure wounds after a surgical operation by anti-inflammatory action.

Regarding wound healing after surgery due to the anti-inflammatory effect of emu oil, Dr. Hobday mentioned: "This is very obvious in transplant operations of a coronary artery when the surface of the foot where the vein has been removed was affected. Following my experience, local emu oil massage two or three times a day accelerated the healing process and reduced the size of scars. "

\subsection{Use of Emu Oil to Treat Microbial, Fungal, and Viral Infections}

Due to its bacteriostatic, anti-fungicidal and anti-viral properties, emu oil is used in numerous formulations to treat infections.

Maurine Pearson [6] patented new emubased formulations that are analgesic, antipruritic and have the property of treating microbial and fungicide infections. They can be used in hospitals to treat wounds, burns and other dermatological problems, as well as to relieve pain caused by skin irritation, wounds and burns [6].

The products stimulate the synthesis of deoxyribonucleic acid (DNA) in the epidermis, reduce inflammation and have a lubricating and moisturizing effect.

They are designed to inhibit the pathogens Trichophyton Rubrum, Microsporum Equinum, Trichophyton Equinum, Microsporum Gypseum, Microsporum Canis, Epidermophyton Floccosum, Trichophyton Mentagrophytes.

The new formulations also inhibit microbial activity caused by Escherichia coli, Salmonella typhimurium, Pseudomonas aeruginosa, Staphylococcus aureus, Enterococcus faecalis, Bacillus Cereus, Candida albicans, Streptococcus agalactiae.

\subsection{Use of Emu Oil to Relieve Arthritis Symptoms}

Since emu oil has anti-inflammatory properties and arthritis is associated with inflammation, the topical application of oil or the ingestion of capsules containing emu oil, may diminish inflammation.

A preliminary study by Dr. Leahey of the Oklahoma Clinic, to determine the effect of emu oil on arthritis-affected hands has indicated that the oil can substantially reduce the pain caused by this disease [7].

In this 2-weeks study, 7 of the 12 emutreated patients reported a significant reduction in pain, swelling of the hand, and stiffness. Of the 8 patients who received the mineral oil instead of emu oil, only one reported the same results.

A more complete study entitled "The Effect of Emu Oil on Osteoarthritis in the Hand" was conducted by Drs. Melanie Cameron and Ray Power at Victoria University, Australia. Its objective was to determine the effect of the emu oil administered locally or ingested, on the force of the squeeze and the sensitivity of the hands affected by osteoarthritis [8].

In this 8-weeks study, 120 volunteers aged 47 to 87 who were diagnosed with osteoarthritis at the hands were initially recruited.

Patients were divided into three groups that had the following treatments:

A. Oil applied locally on both hands (twice daily, morning and evening, for a maximum of $30 \mathrm{sec}$.)

B. Ingested oil ( $5 \mathrm{ml}$, twice a day)

C. Local oil and ingested oil.

The following clinical and functional parameters were determined at the beginning, middle and end of the study: the pain, the joint sensitivity, the pain force, the perception of pain reduction,

The results suggest that emu oil applied locally or ingested can be used to reduce the pain caused by hand osteoarthritis. Due to the fact that a pain relief was found after the third week of the study, the authors suggest that the product should be administered continuously over a longer period to show its therapeutic effect. It has also been mentioned that local application of the oil may be a more effective 
treatment for osteoarthritis if the patient is simultaneously given glucosamine or capsaicin, either locally or orally (3 months, 1 $500 \mathrm{mg}$ daily).

Novartis Pharmaceuticals Pty Ltd in Australia financed the clinical trial "Randomized phase II placebo-controlled study to evaluate the efficacy of topical pure emu oil for joint pain related to adjuvant aromatase inhibitor use in postmenopausal women with early breast cancer" JUST (Joints Under Study) [9].

The objective of this study realized at Mount Hospital, Perth, was to evaluate the efficacy of emu oil to relieve joint pains in menopausal patients who are treated with "aromatase" inhibitors for breast cancer in an early phase. The inhibitors have the role of preventing cancer reappearance, but they cause joint pain (anthralgia) and stiffness as a side effect.

The conclusion of the study performed on 75 patients indicated that arthralgias, as a result of aromatase inhibitor use, may be ameliorated by the use of topical oil massaged onto the joint.

Emu oil was also one of the active ingredients used by Mallozzi and Fallon for the treatment of inflammatory and noninflammatory arthritis [10]. Along with the oil, other ingredients were arnica, calendula, glucosamine, salicylic acid, hamamelis and yam. The medicine was administered as an ointment, cream, gel, emulsion, or liquid solution that is applied directly to the affected area or soaked in a buffer that is held over the area with an adhesive. The emu oil was used as a transdermal delivery agent and antiinflammatory agent.

\subsection{Use of Emu Oil in Lowering Cholesterol}

Dr. Fein and collaborators are the authors of two patents titled "Therapeutic Uses of Emu Oil". The patent claims the discovery that "emu oil can be used therapeutically to lower cholesterol, triglycerides and lowdensity lipoproteins, and to increase high density lipoproteins; prevents and treats allergies; treats headaches; prevents bleeding of the nose; reduces the pain caused by the menstrual cycle " [11].
The inventors state that emu oil can be ingested at least once a day to lower cholesterol. The amount of oil required depends on the patient's cholesterol level, age and weight. Some patients may receive treatment if they ingest between $2-10 \mathrm{ml}$ of oil per day, for the other patients the maximum dose required was $7 \mathrm{ml}$ per day. Generally, a dose of one teaspoon of emu oil per day was effective. The oil may be administered in liquid form or in gelatin capsules.

The following example was described in the patent:

A 60-year-old woman ingested 7-10 drops of oil, or about one teaspoon a day. The patient was previously treated for decreasing cholesterol with Mevocore, but the drug had side effects, causing hair loss. Emu oil has no side effects and the patient's hair has recovered. The results of the analyses are presented in Table 3.

Table 3. Values of the total cholesterol, LDL, HDL, and triglycerides before and after treatment with emu oil

Total Cholesterol, LDL, HDL, Triglycerides

\begin{tabular}{|c|c|c|}
\hline & $\mathrm{mg} / \mathrm{dl}$ & \\
\hline niti & 292 & 40 \\
\hline After 6 months & 264 & 38239 \\
\hline
\end{tabular}

\section{5 Use of Emu Oil to Treat Burns}

The American Emu Association has collaborated with Dr. John Griswold of the Texas Tech University Medical Center in Lubbock, USA, to study the emu oil's efficacy in the healing of wounds caused by burns.

Dr. Griswold presented the results of the study "Evaluation of Emu Oil for Lubrication and Treatment of Burns" at a Conference organized by the American Burn Association.

The presentation can be summarized as follows:

The study was conducted to evaluate the emu oil as a lubricant and to limit scar formation following wound healing. Ten patients were evaluated in a "blind" study that lasted at least 6 months. Patients had their own witness by using emu oil for some burns and using a placebo lubricant for other similar burns. Because the study was blind, patients did not know the identity of the products they were using. The application of both lotions 
was done daily. The treatment lasted between 195 and 385 days. At the end of the treatment, significant differences in pigmentation and flexibility of emu-treated scars were observed in comparison to those obtained after placebo treatment, but there were no differences in wound vascularization. Burn photos indicated that emu-treated burnt surfaces healed much better than those treated with placebo.

Currently, it seems that emu oil is recommended by many doctors to treat burns, even in the case of road accident victims. Due to its composition in fatty acids similar to the skin, emu oil favors the growth of a new skin layer, reducing the need for numerous painful transplants.

Dr. Dan Dean, who practiced medicine in two clinics in Mt. Pleasant, Michigan, USA, used the emu oil to treat the first, second and third burns and concluded that the product has beneficial effects on the skin of the patients. The doctor has achieved remarkable results even in very severe, type three burns [12]. Figures 2 and 3 illustrate the progression of a heartburn treated by Dr. Dean with emu oil.

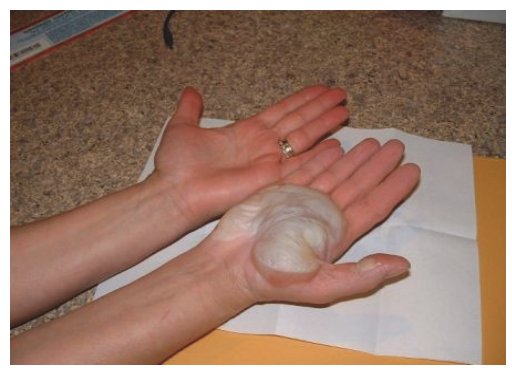

Figure 2. Burn before treatment with emu oil

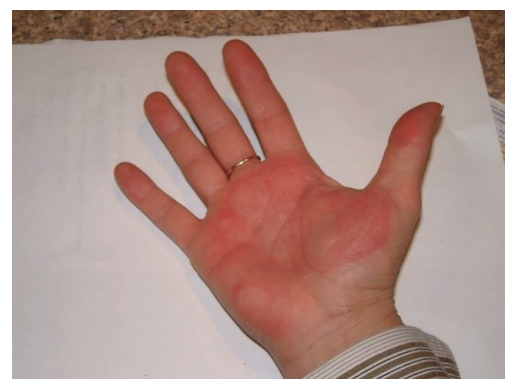

Figure 3. Burn healed after 32 days of treatment

It is known that the treatment of cancer by radiotherapy or chemotherapy can cause burns and inflammation as a side effect, which is manifested in chemotherapy-treated patients by the occurrence of a lot of pains in the mouth (ulcers), which are very painful. As a result of chemotherapy, the layer of cells inside the mouth that is most rapidly divided is affected. After ingestion of emu oil several times a day, patients found a significant reduction in ulceration, resulting in pain reduction.

Promising results have also been obtained in cancer patients treated with radiotherapy. The cancer patients who were to undergo these treatments applied emu oil on the skin 20 minutes before irradiation, or ingested emu oil. The effect achieved was to reduce the intensity of burns, compared to the expected burns from the dose of administered radiation.

\subsection{Use of Emu Oil by Diabetic Patients}

Upper or lower limb wounds of diabetic nature are difficult to heal due to poor blood circulation and can become infected frequently, requiring amputation. Even dry skin can be dangerous for diabetics because it can crack, causing inflammation. Emu oil helps to hydrate the skin, thus preventing inflammation. Moreover, the presence of linolenic and oleic acids in its composition gives the oil anti-inflammatory properties.

The use of emu oil as an insulin transporter in human diabetic patients was patented by Fishman in 2007 [13].

The patent describes formulations of a dermal insulin delivery system composed of emu oil, fatty acid alkyl esters, polyethylene glycol-8 (PEG-8), methylsulfonyl methane (MSM) and a gelling agent.

Henry Schur patented another transdermal application of insulin containing emu oil. External application was done as a patch, without skin irritation being observed in the application area. Prior to the addition of other ingredients, insulin was initially incubated with a biopolymer designed to increase skin permeability of chemically and biologically active substances with a molecular weight greater than 150 Daltons [14].

In 2013 Akram et al. have published a study that refers to the formulation of an insulin emulsion and the evaluation of hypoglycemic activity in an animal model. Eight insulin formulation emulsions were 
prepared using emu oil as a carrier in combination with Carbomer or hydroxypropyl methyl cellulose as a gelling agent and Polisorbate 80 as the emulsifier [15].The biological activity of the emulsion formulation was investigated on Albino rabbits alone and in combination with iontophoresis. The in vivo efficacy of the product was evaluated by measuring the blood glucose level at the start of the experiment and then every 15 minutes for 120 minutes.

The optimal formulation indicated a maximum insulin penetration flow (4.88 \pm $0.09 \mu \mathrm{g} / \mathrm{cm} / \mathrm{hr}$ ) through the mouse skin that was taken. The same formulation was then studied on Albino rabbits. In the first group treated with insulin emulsion only, the blood glucose level increased after 120 minutes, and in the second group treated with insulin and iontophoresis emulsion the glucose level decreased to $125 \pm 5 \mathrm{mg} / \mathrm{dl}$ in 120 minutes.

Insulin absorption through the transdermal emulsion was found to be higher in combination with iontophoresis, resulting in a more pronounced reduction in blood glucose levels. Based on this study, it has been demonstrated that the application of iontophoretic insulin emulsion can be used as an alternative to insulin injection in future studies. In addition, this method is not painful.

\subsection{Dermatological and Cosmetic Usage of EmuOil}

\subsubsection{Skin Hydration}

The moisturizing and cosmetic properties of emu oil have been evaluated in a clinical trial conducted by a team of researchers led by Dr. Alexander Zemtsov, a dermatologist and associate professor at the Indiana University School of Medicine, USA [16].

The ability of the oil to penetrate into the skin and the moisture, as well as its smell, have been compared with the similar properties of a synthetic mineral oil commonly used in the USA as emulsifying agent and lubricant in external cosmetics and pharmaceuticals.

Volunteers selected to participate in the study had normal skin without acne or eczema. The protocol provided that the clinical trial was "blind", meaning that both the patients and the investigators could not initially identify the type of oil that was used by the subjects. In the study the participants were asked to apply an oil on the face and on the body twice a day, for two weeks. After this time, the subjects were examined to detect signs of skin irritation or acne, and then they were recommended to apply the second oil under the same conditions.

At the end of the study, each patient completed an assessment questionnaire using a scale of 0 (unsatisfactory) to 5 (excellent) to compare the effect experienced after applying each oil. They also had to compare moisturizing properties, skin penetration power, texture and any side effect (pore clogging, odor, irritation, etc.) The results presented in Table 4 indicate that emu oil had a global assessment and skin penetration / permeability superior to mineral oil.

Table 4. Evaluation of oils by participants in the clinical trial

Emu oil Mineral oil (average scores)

$\begin{array}{lrr}\text { Overall oil evaluation } & 5 & 3 \\ \text { Texture } & 4 & 3 \\ \text { The ability to penetrate into5 } & 3 \\ \text { the skin / permeability } & \\ \begin{array}{l}\text { Moisturizing properties } \\ \text { Mon }\end{array} & 4\end{array}$

According to the answers given in the questionnaire, all participants in the study $(100 \%)$ mentioned that they preferred emu oil. None of the oils irritated the skin. The use of emu oil in cosmetics is also due to its properties to be an excellent emollient and a good emulsifier. It can replace in cosmetics some commonly used commercially available emollients, which can cause skin irritation and are corrosive. Because it does not contain water or protein, emu oil has the advantage of not creating an environment favorable to the development of bacteria or fungi. Many cosmetics cause allergies or other side effects due to the formation of infectious bacteria.

Emu oil stimulates the regeneration of the skin cells, attenuates the wrinkles, heals the cracks of the skin and protects it from the effects of cold or heat.Currently many emu oil cosmetics are marketed: soaps, creams, lotions, gels. 


\subsubsection{Scars. Transdermis Treatment}

The property of emu oil to reduce or even contribute to the complete disappearance of scars has recently been confirmed by a clinical trial conducted for 3 years by plastic surgeon J. Chao, director of Plastic Surgery Trauma at Albany Medical College, USA [17].

At a preliminary stage, Dr. Chao conducted a pilot study on a small number of patients who had breast reduction surgery. After surgery, on the scar of a breast, the Transdermis Scar Therapy product, which is a serum containing pure emu oil, was applied, and on the second symmetric scar on the second breast was used the Mederma product, currently recommended for the treatment of scarring. Mederma is an onion extract product marketed by Merz Pharmaceuticals.

Treatment of scars with the two products was initiated two weeks after the surgery, when the bandages were withdrawn. After 2 months of treatment, patients reported a more significant reduction in the emu-treated scar compared to Mederma. After this time, all participants in this study requested that the treatment be continued with emu oil. A "blind" observer, who did not know the type of product applied on each breast, came to the same conclusion. Scars treated with emu oil have become less red, thinner, less noticeable, and the skin has lost its stiffness.

Due to the positive results of this limited study in a limited number of patients, Dr. Chao conducted a clinical study on a larger number of patients who underwent surgery. The purpose of this first study to evaluate scar treatment for humans was to clinically prove the efficacy of the emu oil. Following the results of this 3-year clinical trial, Transdermis Scar Therapy, based on emu oil, is used successfully for the treatment of scars caused by injuries, bruises, and surgery.

Figures 4 and 5 shows the development of a patient's scars, following the treatment with Transdermis Scar Therapy. Emu oil also accelerates the healing of bruises and contusions.

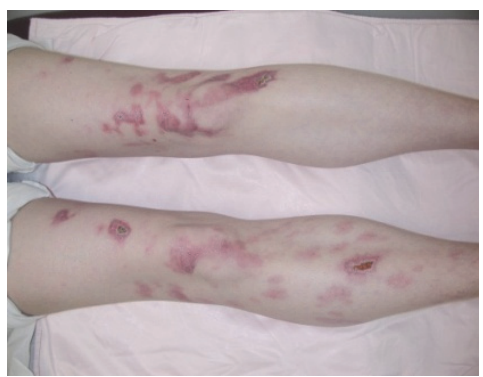

Figure 4. Scars before the treatment

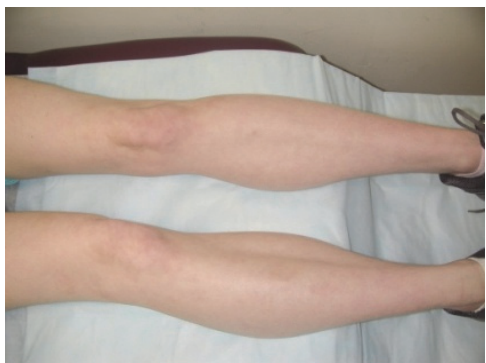

Figure 5. Scars after the treatment

\subsubsection{Eczema}

In recent years, emu oil has begun to be successfully used in the treatment of various types of eczema such as atopic, contact, seborrheic eczema, etc. Due to its chemical composition consisting of $70 \%$ unsaturated fatty acids and bacteriostatic, hypoallergenic, anti-inflammatory and anti-comedogenic properties, emu oil provides skin with nutrients, moisturizes and reduces irritation.

Due to the hypoallergenic properties of emu oil and the absence of phospholipids, emu oil is recommended for topical application on all skin types. Treatment should be repeated two or three times a day, depending on the severity of the eczema. In addition, patients may use soaps and lotions containing emu oil to clean irritants and allergens.

Figures $6 \& 7$ show the progression of a severe facial eczema in an elderly patient who has been treated with oral and injectable synthetic drugs (cortisone, antibiotics) for 8 weeks. As no results were obtained, drug administration ceased and emu oil was started [18]. 


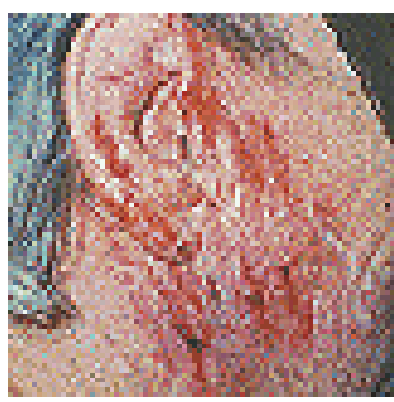

Figure 6. Initial lesion (after conventional treatment)

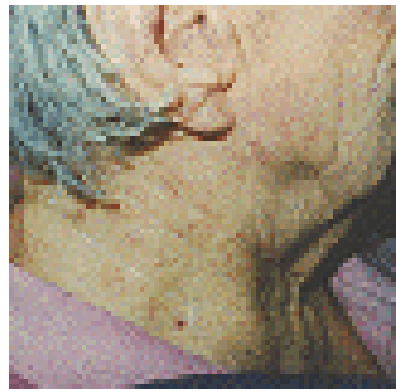

Figure 7. Lesion after 3 weeks of treatment with emu oil

\subsubsection{Psoriasis, Dermatitis}

Because the treatment of psoriasis with steroids has been shown to cause undesirable side effects, alternative treatments based on natural products of plant or animal origin, such as natural oils, tend to be used.

Currently, it cannot be said that emu oil can cure psoriasis, but many people who have used it have seen an improvement in the dry skin condition and the itching caused by this disease. Emu oil nourishes the tissue and can help the normal growth of skin cells, reducing the alternative growth process that causes psoriasis lesions. Currently, however, it is not possible to predict the efficacy of the product for a person affected by psoriasis. The body of each patient being different, the person with psoriasis should test the emu oil to determine its efficacy. Overall, the consumer's conclusions are very positive.

Dr. Jim Kiatos from Victoria University of Technology, Australia, conducted a clinical study on the effect of emu oil on psoriasis [19]. The study aimed to determine whether emu oil administered locally, orally, or by combining the two treatments has an effect on:

- the severity of psoriasis

- the quality of life of patients

- the appearance of the skin surface affected by psoriasis.

In this 12-weeks study, 91 volunteers with a diagnosis of psoriasis vulgaris were recruited.

The severity of psoriasis was evaluated by the Self-Administered Psoriasis Severity Index (SAPSI) before the start of the clinical trial and on the same day of each week throughout the study.

To assess the quality of life, the (Dermatology Life Quality Index,(DLQI) was used. DLQI is a questionnaire containing 10 questions, which refers to patient assessment of symptoms, treatment problems, skin condition and interference with everyday activities. The overall assessment also took into account the doses and frequency of psoriasis medication out of the study.

In conclusion, the results of the clinical trial suggested that emu oil reduced the severity of psoriasis symptoms, such as redness and the scaly appearance of the skin.

Although emu oil is not a cure for psoriasis, the results of the clinical trial have shown that following the use of emu oil, the use of traditional therapy is reduced, which allows the treatment of psoriasis to be cheaper and more effective.

In Romania, Prof. Dr. Maier Nicolae, from the Clinic of Dermatology and Venereology in Cluj Napoca, participated in 2003 in a multinational clinical study titled " The local application of Dr. Michael's cosmetics with therapeutically effect in psoriasis".[20] The study involved researchers from Romania, Hungary, Russia and Austria.

This clinical trial evaluated the therapeutic effect of Dr. Michael's products on psoriasis with varying degrees of severity, as well as the adverse reactions and tolerability of these products. Dr. Michael's products tested in three successive phases were a gel cleansing, a scalp and body ointment, and a skin conditioner, containing emu oil.

In this 8-weeks study, 30 patients with a diagnosis of psoriasis vulgaris were recruited. 
In the morning, on the skin of the body, the cleansing gel was applied and it was massaged on the psoriatic plaques and allowed to act for three minutes. After removing the gel with water, the skin was wiped off and the ointment was applied to the psoriatic plaques, upon which the skin conditioner with emu oil was immediately applied. The operation was repeated in the evening.

In the evening, on the scalp, the psoriatic plaques were massaged with the ointment, and the morning was washed with the cleansing gel. The results of the treatment were clinically recorded on medical visits performed weekly. At the start of the study, three weeks after, and at the end of the study, areas of the patients affected by psoriasis were photographed. Also, psoriasis associated symptoms were quantified during medical visits, using the Psoriasis Area and Severity Index (PASI) score.

The study realized with Dr. Michael's therapeutic cosmetics containing emu oil showed that the products were effective in the treatment of psoriasis, reducing scales in 7 days, tegument infiltration in 20 days, and erythema after 20 days. Following the treatment of scalp lesions, the disappearance of psoriatic plaques occurred after 14 days.

The percentage of cases for which a good and very good therapeutic effect was achieved was $73 \%$.

Similar results were obtained by researchers in Hungary, Russia and Austria. Following the four independent clinical trials involving 121 patients, it was demonstrated that Dr. Michael's preparations are effective for the treatment of psoriasis. Emu oil incorporated in body and scalp balm contributed to the reduction of inflammation and had the role of preventing the loss of flexibility and elasticity of the skin.

Dr. Zemtsov, a professor at the Department of Dermatology, School of Medicine, Indiana University, published the experimental results of a study to test the efficacy of a new topical formulation containing cetirizine in emu oil for the treatment of patients with psoriasis, atopic dermatitis and stasis dermatitis.

Topic dermatitis is an inflammatory skin condition that creates an intense pruritus (itching) with chronic evolution. The disease affects $10-20 \%$ of the population. Stasis dermatitis is manifested by inflammation of the skin caused by slowing or stopping blood circulation in the affected area.

Treatment is done with steroid-based creams, but their prolonged use causes unwanted side effects.

The formula conceived and used in this study is a therapeutic agent that easily penetrates the skin and possesses emulsifying properties. The cetirizine active principle that is dissolved in the emu oil acts as a vehicle that dissolves and transports the ingredient through the skin barrier and has synergistic therapeutic effects with emu oil, or it may be the active pharmaceutical product itself.

The formulation contains the following ingredients: emu oil $(85 \%)$, cetirizine $(2 \%)$, methyl paraben $(0.2 \%)$ which is a conservative agent, and propylene glycol that promotes the solvent and emulsifier properties of emu oil.

The results have shown with certainty that the emu and cetirizine oil have acted in synergy favoring each other their individual therapeutic effects on inflammatory skin diseases. The new anti-inflammatory, antipruritic, moisturizing, topical formulation is very effective for treating atopic dermatitis, psoriasis and other dermatological diseases.

\subsubsection{Cosmetics}

Emu oil is the only natural oil that is easily absorbed into the skin, having the same chemical composition as the oils contained in human skin. The constituents that allow it to penetrate into the skin are monounsaturated fatty acids, also contained in human skin.

Being an efficace transdermal transporter, emu oil has superior moisturizing and nutritive properties compared to other oils used in cosmetic preparations.

Emu oil has the ability to transport vitamins $\mathrm{E}, \mathrm{A}, \mathrm{C}$, ceramide, lipids and other nutrients to skin alleviating the symptoms of skin aging, that are drying, wrinkling and loss of flexibility.

Due to its characteristics, emu oil is used as the main ingredient in many cosmetics, replacing ingredients such as triethanolamine, urea, lanolin, propylene glycol, which may have side effects.

Essential fatty acids that are contained in oil give it the following properties: hydratant, 
ultra penetrating, transdermal transporter, reduces itching, restore the hydrolipid film of the epidermis. Many creams, lotions, gels, containing emu oil are successfully marketed.

\section{Other Uses}

Emu oil is also used for the treatment of acne, rosacea, loss of hair (alopecia), in formulation of antihistaminics, to administrate analgesics and anaesthetics drugs, in the veterinarian field, for protection against insects, for the obtention of an antiserum against poisonous venom of some snakes, and other.

\section{Conclusion}

The therapeutic effects of emu oil for such diversified diseases might astonish.

Dr. Robert Nicolosi, Director of the Center for Health and Disease at the University of Massachusetts, SUA mentioned: "Emu oil is a mysterious medical marvel".

\section{Acknowledgments}

The authors would like to express their great appreciation to Australian Prof. Drs. P. Ghosh, M. Whitehouse, M. Cameron, A. Ferrante, and G. Howarth for sharing with us documentation and results of their scientific research related to emu oil. Their willingness to give us their time so generously has been very much appreciated.

\section{References}

[1] Whitehouse, M.W. Turner AG, Davis CK, Roberts, M.S. Inflammopharmacology, 6(1), (1998), 1.

[2] Craig-Schmidt, M., Brown, A. Smith, P.C., INFORM 6, (1995).

[3] Hopkins, L., Reprinted from AEAB News, Spring 1997 issue.

[4] Sears, B., AEA Annual Symposium,
Rochester, Minnesota, 2000, Emu Today \& Tomorrow, July 2000.

[5] Hobday, G.R., Australian Emu, July/August, (1994) .

[6] Pearson, M., Barr, T., Patent No. 6, 528,040, March 4, (2003) .

[7] Leahey, Emu Today \& Tomorrow, July 1995.

[8] Cameron, M., Power, R., Annals of the Rheumatic Disease 2005, 64, 1570.

[9] Chan, A.et al., Support Care Cancer, 25 (2017), 3785

[10] Mallozzi, O., Fallon J. M. Patent No. US 20070048386 A1, March (2007) .

[11] Fein, E., Caputo, J., Nagal, KL., Patent No. 5,662,882, September 2 (1997) .

[12] Emu Oil: Reexamining a Natural Remedy with Today's Technology, Revised and Updated, edited by Schatz publishing Group, LLC (2004), 26.

[13] Fishman, R., Patent No. US 7,291,591

B2 (2007)

[14] Schur, H., Patent No.US 20070092571 A1.

[15] Akram, M., Naqvi, S.B., Khan, A., Pakistan J. of Pharm. Sciences 26 (2) (2013), 323.

[16] Zemtsov, A., Gaddis, M., MontalvoLugo, V. M., Australasian J. of Dermatology 37 (3), (1996), 159.

[17] Chao, D.J., Doster, C., Plastic Surgery

Practice, March (2006) .

[18] Emu Today \&Tomorrow, October 1998.

[19] Kiatos, J., http:

www.emuspirit.com/why-emuspirit/psoriazistrial.htm [20] Goodskincare by Psoriasis \&Skin Clinic, April 4 (2012) .

[21] Zemtsov, A., Hosier, H, J. of Cosmetics, Dermatological Sciences and Applications, 3 (2013), 18 